\title{
Optical Gas Sensing with Encapsulated Chiral-Nematic Liquid Crystals
}

\author{
Lukas Pschyklenk,* Thorsten Wagner, Alexander Lorenz, and Peter Kaul
}

Cite This: ACS Appl. Polym. Mater. 2020, 2, 1925-1932

Read Online

ABSTRACT: Optical gas sensors based on chiral-nematic liquid crystals ( $\mathrm{N}^{*}$ LCs) forming one-dimensional photonic crystals do not require electrical energy and have a considerable potential to supplement established types of sensors. A chiral-nematic phase with tunable selective reflection is induced in a nematic host LC by adding reactive chiral dopants. The selective chemical reaction between dopant and analyte is capable to vary the pitch length (the lattice constant) of the soft, self-assembled, one-dimensional photonic crystal. The progress of the ongoing chemical reaction can be observed even by naked eye because the color of the samples varies. In this work, we encapsulate the responsive $\mathrm{N}^{*} \mathrm{LC}$ in microscale polyvinylpyrrolidone (PVP) fibers via coaxial electrospinning. The sensor is, thus, given a solid form and has an improved stability against nonavoidable environmental influences. The reaction behavior of encapsulated and nonencapsulated $\mathrm{N}^{*} \mathrm{LC}$ toward a gaseous analyte is compared, systematically. Making use of the encapsulation is an important step to improve the applicability.

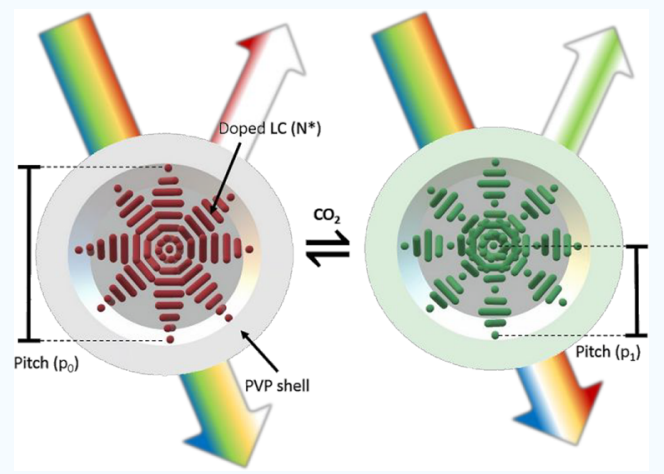

KEYWORDS: liquid crystal, chiral-nematic, tunable pitch, low molecular weight, coaxial electrospinning, encapsulation

\section{INTRODUCTION}

Thermotropic liquid crystals (LCs) are mesophases, which appear in the phase diagram of organic substances, consisting of molecules with a characteristic shape, for example in rodlike (calamitic) substances. The anisotropy leads to unique optical properties, which have been fascinating scientists for about 130 years already. The number of publications dealing with the properties and structures of LCs increased rapidly in the late 1950s when G. H. Brown and W. G. Shaw published their review article "The Mesomorphic State". The first liquid crystal display (LCD) was reported in the late 1960s. ${ }^{2}$ But LCDs were not the only application area. The strong responsiveness of LCs to external influences including heat, light, as well as electric and magnetic fields, combined with an "inherent fragility" 3 of the mesophases makes them suitable for sensory applications. The reaction of the chiral-nematic mesophase to changes in temperature was of particular interest. Fergason et al. investigated the suitability of $\mathrm{N}^{*}$ LCs for temperature measurements in general and in particular for the measurement of skin temperature for medical diagnosis (thermography). ${ }^{4,5}$ The rapid technical progress renders us more and more dependent on reliable sensor technology, today. The energy consumption of sensors becomes more important because of the demand for battery powered, long operating sensor systems, which is driven forward by ongoing developments, such as the rapidly expanding Internet of Things (IoT). ${ }^{6}$ A crucial measurand for these applications is the gas concentration. Many of the well-established systems like semiconductor-based gas sensors, which need to operate at high temperatures, or NDIR type sensors usually have a high power consumption or relatively high system costs. It is, therefore, not surprising that LC-based gas sensors, which indicate the presence of a specific gas by a color change visible to the naked eye and which are not dependent on a power supply, cause great interest. However, there are still unsolved problems, for example, the sensibility of LCs toward mechanical and environmental influences.

The term chiral-nematic indicates that this mesophase has its origin in a nematic mesophase, which becomes chiralnematic by the addition of a chiral, optically active substance. Sometimes the term cholesteric liquid crystal is still used for chiral-nematic LCs, due to historical reasons. The first substances with chiral mesophases discovered were cholesteryl benzoate and cholesteryl acetate. ${ }^{7}$ These liquid-crystal-forming molecules (cholesteryl esters) are intrinsically chiral and show chiral mesophases without the need of a chiral dopant. The chiral-nematic mesophase is characterized by a helical structure. In calamitic LCs, the local mesogen alignment is described by a pseudovector (director). In a $\mathrm{N}^{*} \mathrm{LC}$, the LC

Received: February 10, 2020

Accepted: April 21, 2020

Published: April 21, 2020

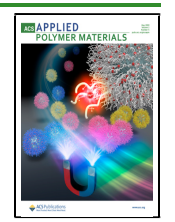


director is twisted continuously along one axis with a pitch length $\mathbf{p}$. The presence of the helical structure leads to a periodic variation of the local refractive index along the helical axis. Such materials (where the pitch length approximately equal to the wavelength of visible light) are well-known as photonic crystals and show selective reflection of light. ${ }^{8}$ In the case of $\mathrm{N}^{*}$ LCs, a one-dimensional photonic crystal is intrinsically formed by self-assembly. A $\mathrm{N}^{*} \mathrm{LC}$ is birefringent. Quite characteristically, only the fundamental diffraction order (selective reflection band) is seen in a $\mathrm{N}^{*} \mathrm{LC}$, and the reflected light is circularly polarized. The location of the maximum of the reflection peak can be calculated by Bragg's law: $\lambda_{\max }=2 \times$ $\cos (\theta) \times \bar{n} \times(\mathbf{p} / 2)$, where $\theta$ is the angle of incidence, $\bar{n}$ is the average refractive index of the $\mathrm{N}^{*} \mathrm{LC}$, and typically has a value of $\bar{n} \approx 1.6$. Although a $360^{\circ}$ twist is required to physically describe one helical pitch of the $\mathrm{N}^{*}$ LC helix, for light traveling along the helical axis, the layer spacing is usually assumed as $\mathbf{p} /$ 2. Accordingly, if $\mathbf{p}$ is in the size range of the wavelength of visible light, the central wavelength of the photonic band gap is in the visible range, too. A change of $\mathbf{p}$ has a direct influence on the wavelength-range of the selective reflection. This change can be achieved for example by adsorbing certain substances on substrate surfaces or dissolving them in the $\mathrm{N}^{*} \mathrm{LC}$, causing a swelling of the helical structure and a redshift of the reflected wavelength. ${ }^{9}$ Since the selectivity of the sensor here is mainly based on the solubility of the analyte in the LC, we assume that a chemical reaction between a reactive dopant and an analyte in the gas phase has clear advantages in selectivity. A reaction of such a dopant with the analyte changes the chemical properties of the dopant and therefore the ability of the dopant to induce the helical structure in the nematic host LC- the helical twisting power (HTP). Mulder et al. concluded in their review ${ }^{9}$ that low molecular weight $\mathrm{N}^{*}$ LCs are the most sensitive and selective LC sensors, but their liquidlike nature makes it difficult for them to reconcile with possible applications. A possible solution for this problem might be the encapsulation of the $\mathrm{N}^{*}$ LC. Kim et al. ${ }^{10}$ suggested the encapsulation of $\mathrm{N}^{*} \mathrm{LC}$ in polymer fibers via coaxial electrospinning. Lagerwall, Scalia, and co-workers ${ }^{11-16}$ have investigated coaxial electrospun LCs encapsulated in PVP fibers with a focus on the alignment of the nematic mesophase and the selective reflection seen in confined $\mathrm{N}^{*}$ LCs. A remarkable observation by Enz et al. ${ }^{15}$ was that the selective reflection even occurs when only a half pitch is confined within the fiber. In contrast, one-dimensional photonic crystals (Bragg-stacks) typically produce a visible selective reflection only if several layers are present. Since these layers each have a thickness of several hundred nanometers, such structures have thicknesses of at least a few micrometers.

Han et al. ${ }^{17}$ investigated a $\mathrm{CO}_{2}$-sensitive $\mathrm{N}^{*} \mathrm{LC}$, where a carbamate forming diamine (Figure S1) was used as chiral dopant and dissolved in the nematic LC E7. In the presence of $\mathrm{CO}_{2}$, the hydrogen bonds between the diamine and TADDOL are broken, allowing $\mathrm{CO}_{2}$ and the diamine to form a carbamate. The mean HTP of the carbamate and the free TADDOL is higher than that of the hydrogen-bonded compound, which leads to a shortening of the pitch and thus to a blue shift in the reflected wavelength. Since the reaction is reversible, flushing with nitrogen or synthetic air leads to the reverse reaction (and accordingly, a red shift of the selective reflection was seen, again).

In the present work, a $\mathrm{CO}_{2}$-sensitive $\mathrm{N}^{*} \mathrm{LC}$ was investigated in coaxial electrospun PVP fibers. PVP was chosen because it allows a relatively large variation of electrospinning parameters. For a better readability, the $\mathrm{CO}_{2}$-sensitive $\mathrm{N}^{*} \mathrm{LC}$ used is simply referred to as $\mathrm{N}^{*} \mathrm{LC}$ in this work from here on. The influence of encapsulation on the sensitivity of the sensor was investigated, and the created fiber shaped sensor material was characterized. The term sensitivity in this case is used to describe the shift of the reflection maximum as a result of the chemical reaction between the dopant and the analyte.

\section{EXPERIMENTAL SECTION}

Preparation of $N^{*}$ LC. The $N^{*}$ LC was prepared by adding 0.263 $\mathrm{g}$ of an equimolar mixture of $(1 R, 2 R)-(+)$-1,2-diphenylethylenediamine (Sigma-Aldrich) and a TADDOL derivate $((4 R, 5 R)-2,2-$ dimethyl- $\alpha, \alpha, \alpha^{\prime}, \alpha^{\prime}$-tetra(2-naphthyl)dioxolane-4,5-dimethanol, Sigma-Aldrich) to $5 \mathrm{~g}$ of the nematic liquid crystal mixture E7 (Synthon Chemicals). The two chiral substances form a hydrogen bonded compound, which is used as the reactive chiral dopant (Figure S1). The mixture was heated to the isotropic phase at $70{ }^{\circ} \mathrm{C}$ and stirred until the chiral dopant was completely dissolved. The mixture was subsequently allowed to cool down to ambient temperature and stored in a glass vessel.

Coaxial Electrospinning. To prepare the polymer solution for the fiber shell $20 \mathrm{~g}$ of PVP $\left(M_{\mathrm{w}} \sim 1300000 \mathrm{~g} / \mathrm{mol}\right.$, Sigma-Aldrich) were dissolved in $80 \mathrm{~g}$ of ethanol (Carl Roth). The coaxial needle (Linari irl) was connected via polyetheretherketone (PEEK) capillaries (ID $0.75 \mathrm{~mm}$ ) to two syringe pumps (LA110 Landgraf). The material PEEK was chosen to minimize pressure fluctuations, which can occur by expansion and contraction of the capillaries. The $\mathrm{N}^{*} \mathrm{LC}$ was pumped with a flow rate of $20 \mu \mathrm{L} / \mathrm{min}$, while the PVP solution was pumped with $30 \mu \mathrm{L} / \mathrm{min}$. The distance between the coaxial needle and the collector electrode was $25 \mathrm{~cm}$. The collector electrode had a high negative electric potential of $-7 \mathrm{kV}$ and the needle had a high positive electric potential of $12 \mathrm{kV}$. The diameters of the inner needle were $0.83 \mathrm{~mm} / 0.51 \mathrm{~mm}(\mathrm{OD} / \mathrm{ID})$ and of the outer needle $1.83 \mathrm{~mm} / 1.37 \mathrm{~mm}$ (OD/ID). Both needles have a length of $10.9 \mathrm{~mm}$. The collector electrode was ring-shaped to collect freely hanging fibers and prevent fiber deformation by colliding with the electrode. The electrospinning setup with the ring-shaped collector electrode is shown in Figure S2 the right-hand side.

\section{METHODS}

Pitch Determination. The pitch of the $\mathrm{N}^{*} \mathrm{LC}$ at ambient temperature was determined by the Grandjean-Cano method using a plano-convex lens ${ }^{18}$ with a radius of curvature of $7.7 \mathrm{~mm}$. The $\mathrm{N}^{*} \mathrm{LC}$ was dropped on a poly(vinyl alcohol) (PVA, $M_{\mathrm{w}} \sim 10000$, SigmaAldrich) coated and uniaxially rubbed microscope slide. The planoconvex lens was placed on the LC film obtained with the convex side pointing down (Figure 1). The Cano lines were observed in a digital polarized optical microscope (Keyence VHX-600) in reflectance mode and with crossed polarizers. The pitch $\mathbf{p}$ was calculated by measuring the radii of two adjacent lines: $\mathbf{p}=\Delta r^{2} / R$, with $\mathbf{p} / 2=\left(d_{n}-\right.$ $\left.d_{n-1}\right)$ and $d \ll 2 R$.

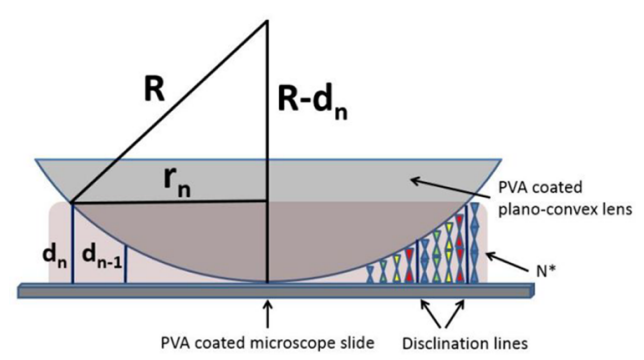

Figure 1. Schematic of the pitch determination by Grandjean-Cano method, where $R$ is the lens curvature radius, $r$ the measured radius of the Cano line, and $d_{n}$ the local thickness of the $\mathrm{N}^{*} \mathrm{LC}$. 
Temperature Dependency of the Selective Reflection Band. The temperature dependency of the selective reflection band was investigated by measuring reflectance spectra with a fiber optic spectrometer. A film of the $\mathrm{N}^{*} \mathrm{LC}$ was prepared by spreading a droplet of the $\mathrm{N}^{*} \mathrm{LC}$ on the surface of a microscope slide with a spincoater (Spin150, SPS). The sample obtained was placed on a microscopy hot stage (Linkam) and heated from $5{ }^{\circ} \mathrm{C}$ in steps of $5{ }^{\circ} \mathrm{C}$ to a temperature of $50{ }^{\circ} \mathrm{C}$ with a holding time of $5 \mathrm{~min}$ at each step and a recording interval of five seconds. The reflectance spectra of the sample were recorded in the visible wavelength range with a fiber optic spectrometer ((Ocean Optics Spectrometer, Flame S-VIS-NIRES, $\delta \lambda \approx 1.5 \mathrm{~nm}$ ) by using a Y-fiber (Premosys fiber-bundle, $1.8 \mathrm{~mm}$ ). The peak maximum was determined from the recorded reflectance spectra after smoothing by a Savitzky-Golay filter with window size of 200 data points and polynomial order of 2 .

Differential Scanning Calorimetry (DSC). The influence of encapsulation on the phase transition temperature was investigated with differential scanning calorimetry (DSC 214 Polyma). Two types of coaxial spun fibers, one filled with E7 and one filled with the $\mathrm{N}^{*}$ LC, were investigated, respectively. Uniaxial spun PVP-fibers served as reference. Samples of a mass between 5.8 and $19.4 \mathrm{mg}$ where filled into crucibles with pierced lids and heated with a heating rate of 10 ${ }^{\circ} \mathrm{C} \mathrm{min}^{-1}$ from -10 to $200{ }^{\circ} \mathrm{C}$. The final temperature was held for 5 min and subsequently; the samples were cooled to $-10{ }^{\circ} \mathrm{C}$ again with a cooling rate of $10{ }^{\circ} \mathrm{C} \mathrm{min}{ }^{-1}$ and heated up again to $200{ }^{\circ} \mathrm{C}$ with 10 ${ }^{\circ} \mathrm{C} \mathrm{min}^{-1}$. The measurement was conducted in nitrogen atmosphere (flow rate of $40 \mathrm{~mL} \mathrm{~min}{ }^{-1}$ ) to avoid any oxidative degradation.

Comparison of Sensitivity and the Shift-Rates of Nonencapsulated and Encapsulated $\mathbf{N} *$ LC. With respect to the comparison of encapsulated and nonencapsulated $\mathrm{N}^{*} \mathrm{LC}$, a measuring chamber was constructed, which allows recording the optical response of different sample shapes in defined gas atmosphere (Figure 2a).
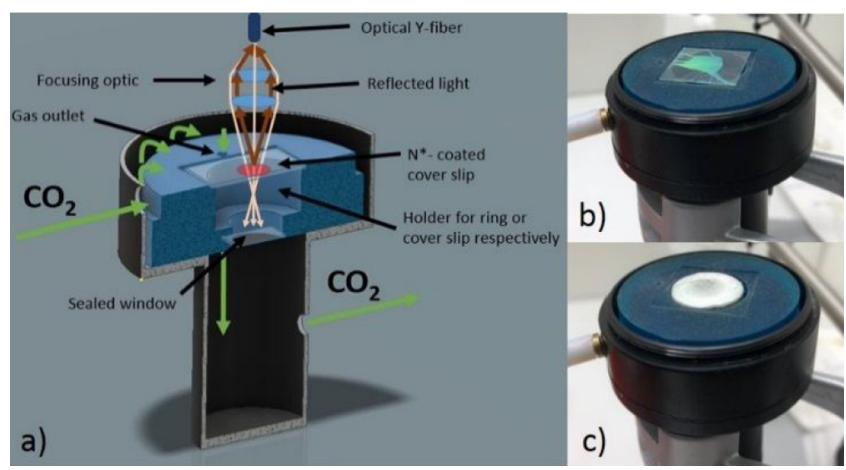

Figure 2. (a) Schematic UV-vis measuring chamber. (b) Photograph of $\mathrm{N}^{*}$ LC coated microscope coverslip. (c) Photograph of an encapsulated sample (ring-shaped electrode covered with coaxial fibers) placed on the sample holder.

Films of the $\mathrm{N}^{*} \mathrm{LC}$ were prepared on a PVA-coated and uniaxially rubbed microscope coverslip (Figure 2b) using a spin-coater (Spin150,SPS) at $3000 \mathrm{rpm}$ for $70 \mathrm{~s}$. These samples are called nonencapsulated in this work. The coaxial fibers deposited on the ring-shaped electrode are called encapsulated samples (Figure $2 \mathrm{c}$ ). For the spectra recorded in both sample types, the reflectance spectrum of a silicon wafer was used as reference due to a better comparability between the two sample types and the well-known reflectivity of silicon. The measurement chamber was closed and flushed with either nitrogen or synthetic air containing a defined partial pressure of $\mathrm{CO}_{2}$ gas by using two mass-flow-controllers (MFC, MKS). In every case, the total gas flow was $200 \mathrm{~mL} \mathrm{~min}^{-1}$. To obtain the maximum signal to expect the $\mathrm{CO}_{2}$ concentration was switched alternately between $0 \%$ and $100 \%$, which gave the best and most reliable data. In a first experiment, fast gas atmosphere changes in the interval of $1 \mathrm{~min}$ were made to obtain information on sensitivity and the shift-rate. In a second experiment, long intervals of $2 \mathrm{~h}$ have then been investigated under the assumption that both the forward and the reverse reaction were completed.

Measurements of the Refractive Index. The mean refractive index $\bar{n}$ was measured with an Abbe refractometer (Krüss, AR4), while $n_{\|}$was measured with a digital refractometer (Krüss, DR6100) using only one prism and total reflection. In both cases the $\mathrm{N}^{*} \mathrm{LC}$ was applied on the prism and the refractive index was measured at a temperature of $20.0^{\circ} \mathrm{C}$. The $\mathrm{N}^{*} \mathrm{LC}$ was when flushed with $\mathrm{CO}_{2}$, until a clear color change was observed, and the measurement of the refractive index was repeated.

\section{RESULTS AND DISCUSSION}

Properties of $\mathrm{N} *$ LC. The Grandjean-Cano measurement of the as prepared $\mathrm{N}^{*} \mathrm{LC}$ showed regular ring-shaped Cano lines (Figure 3a), which clearly indicated the formation of the

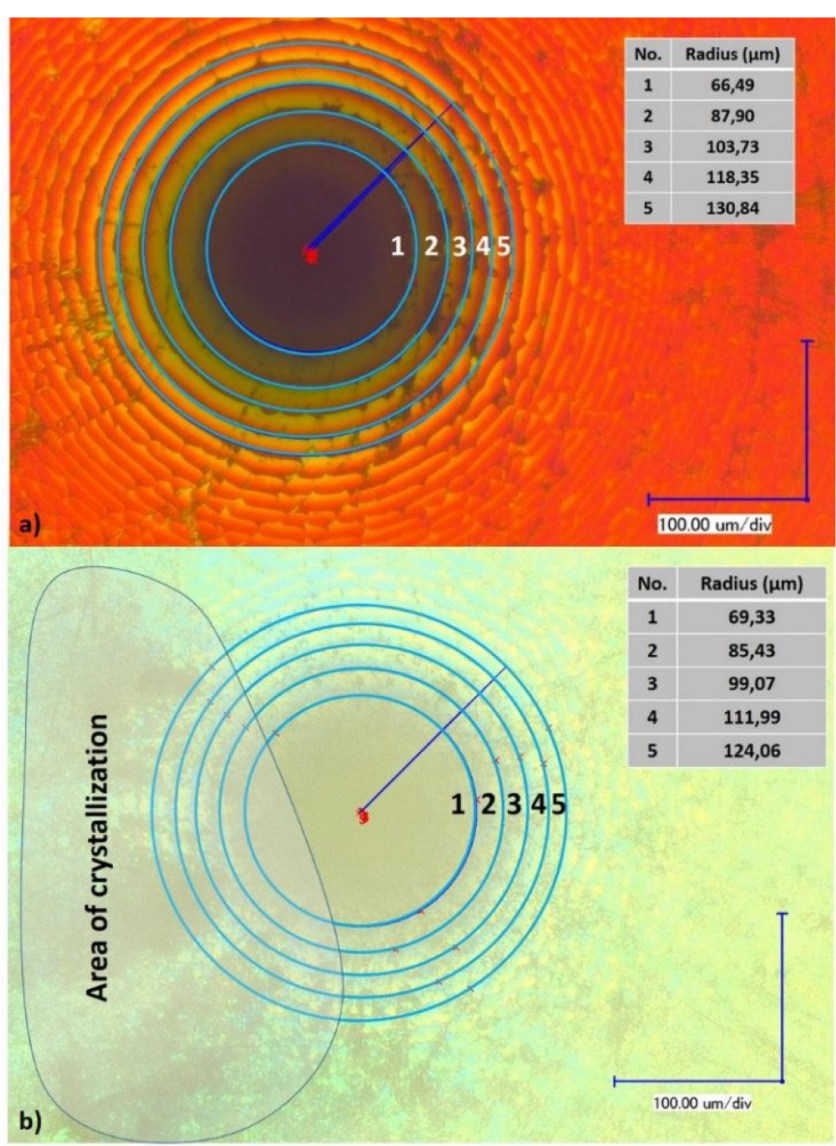

Figure 3. Microscope image of the Cano lines/rings of a nonencapsulated sample before (a) and after (b) reaction with $\mathrm{CO}_{2}$; the ring radii are used for pitch determination.

chiral nematic phase. A pitch of $412( \pm 16) \mathrm{nm}$ was calculated by using the determined mean refractive index $\bar{n}=1.637$. With $\Delta \lambda=\Delta n \times p$, the width $\Delta \lambda^{19}$ of the reflection band was estimated as $\Delta \lambda=87.3( \pm 3.4) \mathrm{nm}$, and the position of the reflection peak maximum was estimated as $675( \pm 26) \mathrm{nm}$. The values obtained fit well to the color impression of the microscope image (Figure 3a), as well as to the actual observed reflection spectrum (Figure $4 \mathrm{a}$ ) of the nonencapsulated sample. Before the reaction with $\mathrm{CO}_{2}$, the reflection peak maximum was located at $665( \pm 2) \mathrm{nm}$. To investigate if the sample properties were affected by the reaction, a GrandjeanCano measurement was conducted while the $\mathrm{N}^{*} \mathrm{LC}$ was 

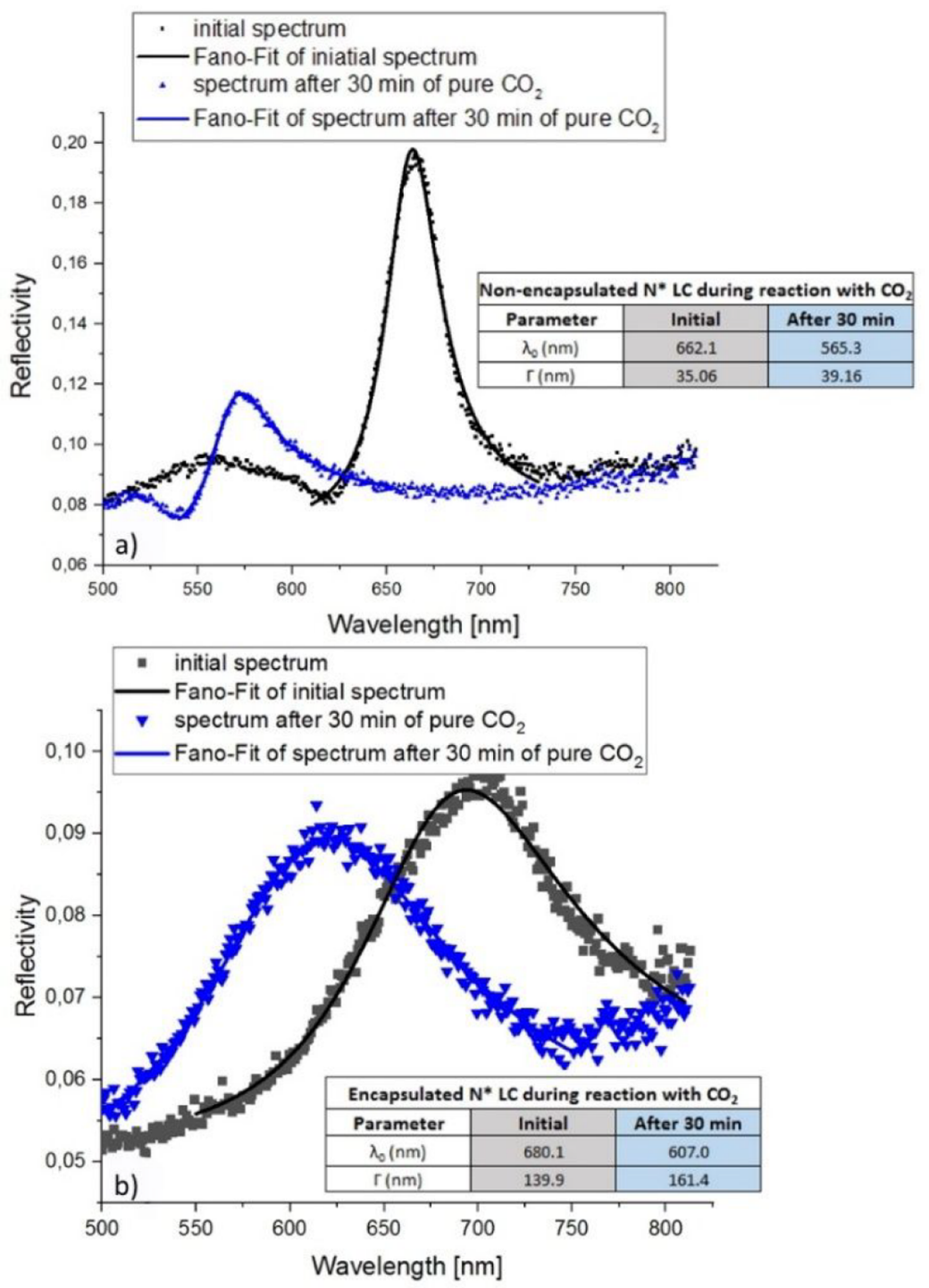

Figure 4. (a) Reflection spectra of nonencapsulated sample before (black line) and after (blue line) reaction with $\mathrm{CO}_{2}$. (b) Reflection spectra of encapsulated sample before (black line) and after (blue line) reaction with $\mathrm{CO}_{2}$.

exposed to pure $\mathrm{CO}_{2}$. A color change towards shorter wavelengths which first affected the film edge and then propagated towards the film center could be observed during this experiment. The propagation speed of the well-visible reaction front progressively decreased and almost came to a standstill when the first visible Cano rings were reached. By carefully tapping on the measuring chamber the color did change abruptly. Microscopic pictures of this experiment can be found in Figure S3. The pitch calculated using the radii shown in Figure $3 \mathrm{~b}$ decreased to a value of $350( \pm 18) \mathrm{nm}$, which corresponded to a calculated reflection peak maximum at $573( \pm 29 \mathrm{~nm})$. This result fits well to the measured reflection peak maximum shown in Figure 4a. Because of the asymmetric peak shape, which is probably caused by additional Mie scattering, Fano fit was used for the evaluation

$$
F(\lambda)=A_{0}+\frac{F_{\max }}{\left(1+q^{2}\right)} \chi \frac{\left[\frac{q+2\left(\lambda-\lambda_{0}\right)}{\Gamma}\right]^{2}}{1+\left[\frac{2\left(\lambda-\lambda_{0}\right)}{\Gamma}\right]^{2}}
$$

with $F_{\max }$ being the maximum intensity, $q$ the asymmetry factor, $\lambda_{0}$ the wavelength at $F_{\max } A_{0}$ the intensity offset, and $\Gamma$ the peak width. The fitted line shapes are seen in Figure 4 as solid lines. The data extracted from the experiment showed that the peak width hardly changed in the course of the reaction, which means there is no change in the refractive index and thus, the helical pitch changed, selectively. This tendency (constant refractive indices) was also supported by in situ measurements of the refractive index with two different refractometers using transmitted light and reflection methods. The measured refractive indices $\bar{n}=1.637$ and $n_{\|}=1.531$ remained constant before and after contact with pure $\mathrm{CO}_{2}$. Both values were slightly higher, than expected from literature $\left(\bar{n} \approx 1.62, \mathrm{E} 7,20{ }^{\circ} \mathrm{C}\right) .^{20}$ The reason for this is most probably the solved dopant. Another observation is an increased precipitation of an undetermined substance (left side of Figure 3b) disturbing the Cano line formation.

Figure $4 \mathrm{a}$ shows the reflection spectra of a nonencapsulated, and Figure $4 \mathrm{~b}$ shows the reflection spectra of an encapsulated sample. Although the reflectivity is lower and the peaks are wider, there was a clear blue-shift in the same order as 
observed using the nonencapsulated sample. The results show that both, the nonencapsulated and the encapsulated $\mathrm{N}^{*} \mathrm{LC}$, can be utilized as an optical gas transducer for detection of $\mathrm{CO}_{2}$.

Temperature Dependency of the Pitch Height. In addition to the dependency of the pitch height on the reaction with $\mathrm{CO}_{2}$ investigated here, there was also a dependence on the temperature. Therefore, the temperature must be kept constant during the measurement or the pitch change at the measured temperature must be known exactly. The reflection peak maximum was shifted to higher wavelength with increasing temperature. The shift of the reflection peak maximum of one encapsulated and one nonencapsulated sample during the temperature program is shown in the Supporting Information (Figure S4). The last two steps at 45 and $50{ }^{\circ} \mathrm{C}$ were already in the infrared wavelength range. Kuball et al. ${ }^{21}$ investigated the temperature dependence for different TADDOL-dopants and found an increasing helical pitch in most cases when temperature was increased. However, the host LCs in these investigations were ZLI-1695 and K15.

Properties of the Coaxial Electrospun Fibers. The reflection peak maxima of the encapsulated samples were located at slightly higher wavelengths $(668-700 \mathrm{~nm})$, which corresponded to a pitch of 408-428 nm. Most likely, ethanol, which was used as solvent in the electrospinning process, was dissolved also in the $\mathrm{N}^{*} \mathrm{LC}$ when both substances met at the exit of the coaxial needle. The presence of a minuscule amount of ethanol as solvent within the crystal structure increased the mean distance between the mesogens and lead to an increased pitch. The fibers obtained had outer diameters of 6-10 $\mu \mathrm{m}$ and inner diameters of $2-5 \mu \mathrm{m}$, and were well and evenly filled with the $\mathrm{N}^{*} \mathrm{LC}($ Figure 5). Within each fiber, there was a blue

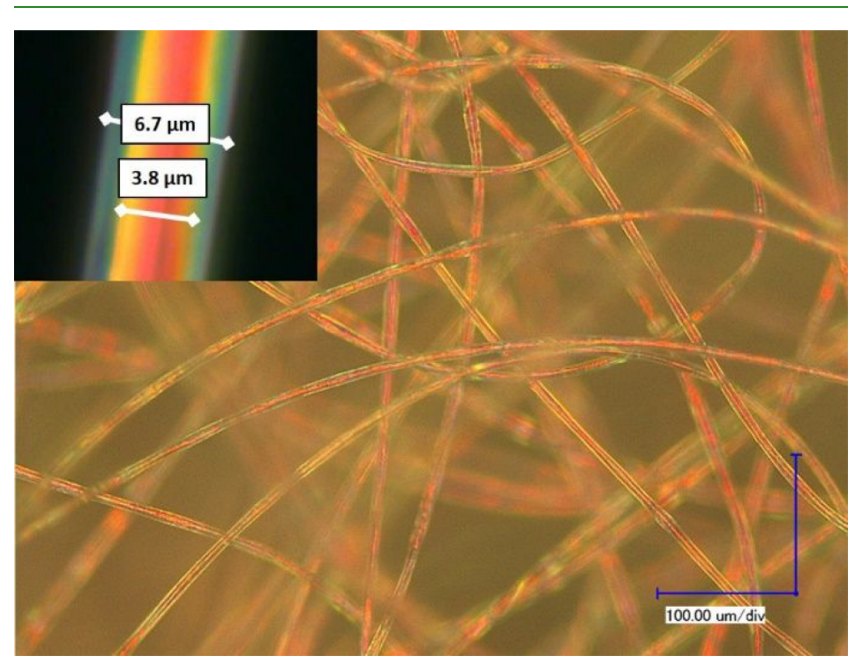

Figure 5. Microscopic picture of the coaxial electrospun fibers.

shift of the color seen near the fiber edge, which was caused by the cylindrical shape and can be understood by Bragg's law. This observation is consistent with the results of Lagerwall et al. ${ }^{11}$ and Enz et al., ${ }^{14}$ who were able to show by polarized Raman spectroscopy and X-ray scattering that the helical axis must be perpendicular to the fiber axis. The reflection peaks recorded in the fiber samples were therefore wider than in the flat, nonencapsulated $\mathrm{N}^{*}$ LC films. The intensity of the reflection spectra, on the other hand was almost independent of the viewing angle. This effect is also seen in Figure 5:
Despite their various spatial orientation, the fibers show the same reflection colors.

Differential Scanning Calorimetry. The measurements of the PVP-fibers compared to the measurements of the coaxial PVP-E7 fibers showed that the LC had a strong softening effect. For the PVP-fibers, a glass transition at $183^{\circ} \mathrm{C}$ was seen, (Figure S5a) which is slightly higher than expected from the literature $\left(174{ }^{\circ} \mathrm{C}\right) .^{22}$ This might be explained by the low contact area between the fibers and the crucible. The wide endotherm peak with a maximum of $118{ }^{\circ} \mathrm{C}$ belonged to hydrogen bonded water. PVP is soluble in water and fairly hygroscopic. Beside the nematic-isotropic transition at 60-61 ${ }^{\circ} \mathrm{C}$, PVP-E7 fibers showed an endotherm peak at $78{ }^{\circ} \mathrm{C}$ (Figure 6), which fits to the boiling point of ethanol that

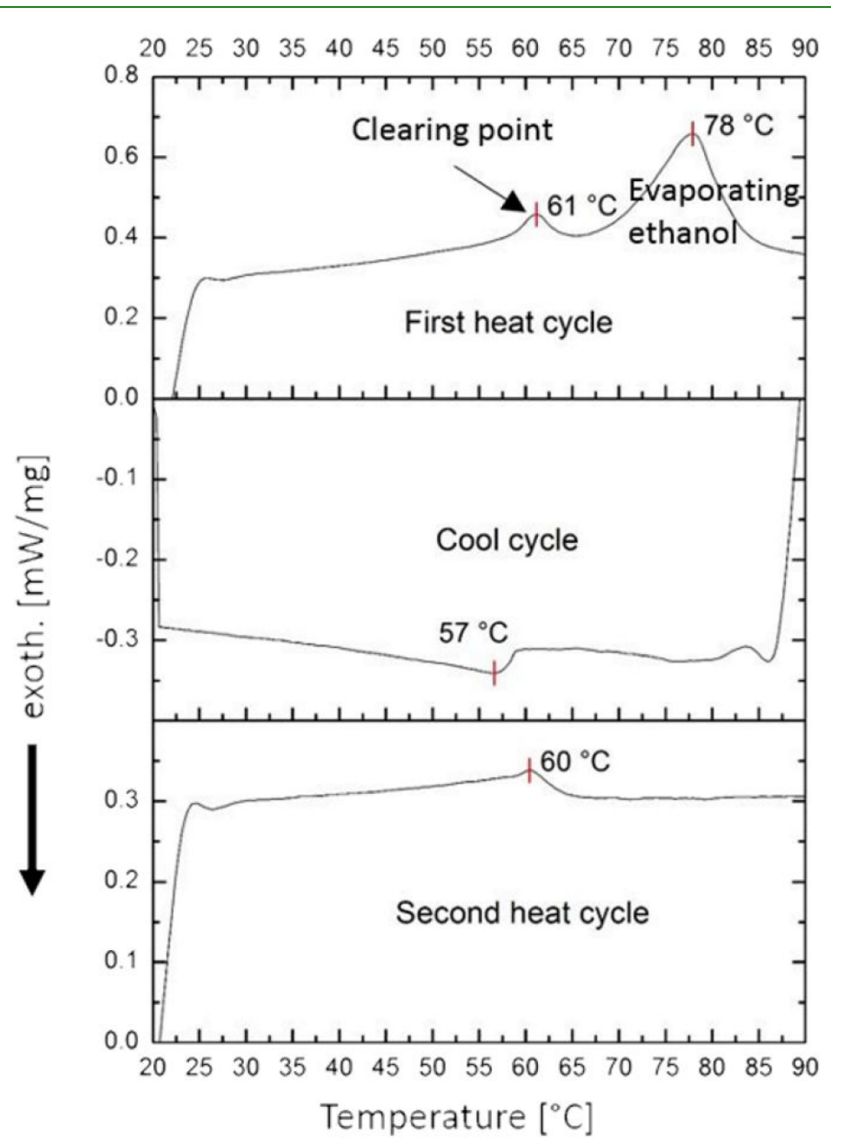

Figure 6. DSC traces of coaxial PVP-E7 fibers $\left(10^{\circ} \mathrm{C} \mathrm{min}^{-1}\right)$.

remained in the fibers during the electrospinning process. Opening the crucibles after the DSC analysis showed that the PVP-E7 fibers were completely liquefied (Figure S5c) between 70 and $80{ }^{\circ} \mathrm{C}$, while drops of $\mathrm{E} 7 \mathrm{can}$ be observed on solidified PVP-plates unequally distributed on the bottom of the crucible. A similar DSC-signal was seen, when the $\mathrm{N}^{*} \mathrm{LC}$ instead of E7 was used as core material (Figure S5b). The electrospun fibers were liquefied in the same temperature range. Uniaxial PVP-fibers were temperature stable until at least $200{ }^{\circ} \mathrm{C}$.

Comparison of Sensitivity and Shift-Rate of Nonencapsulated and Encapsulated N* LC. The maximum spectral shift during the reaction with $\mathrm{CO}_{2}$ was determined: A maximum shift indicated a full conversion of the dopant. Thus, a long-term measurement was conducted by alternating synthetic air and $\mathrm{CO}_{2}$ every $2 \mathrm{~h}$. At the beginning of the 
experiment, the peak position was located at $664 \mathrm{~nm}$ (Figure 7) for the nonencapsulated $N^{*}$ LC, which corresponded to a

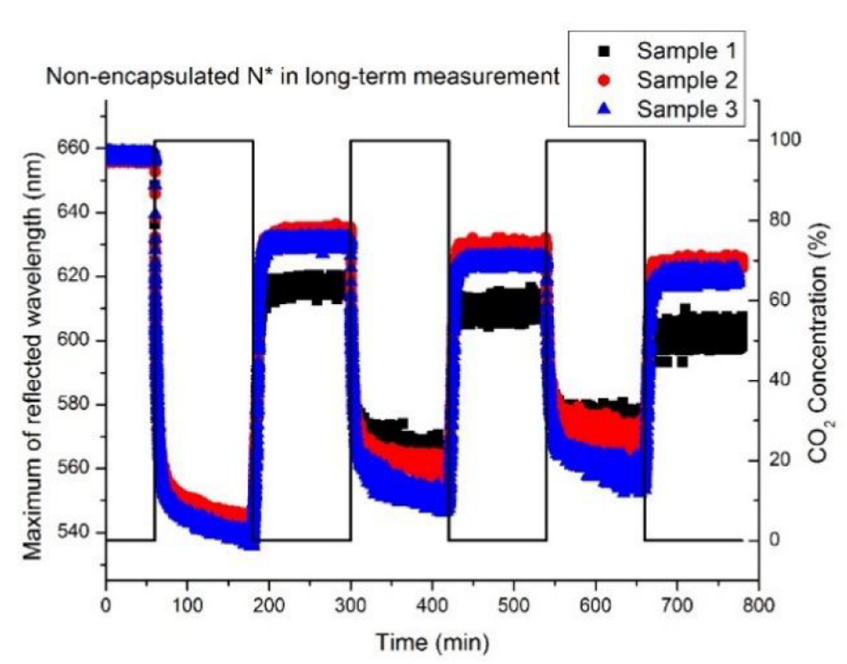

Figure 7. Long-term measurement of the nonencapsulated sensor with $\mathrm{CO}_{2}$ and synthetic air.

HTP value of $+154 \mu \mathrm{m}^{-1}$. Upon repeated measurements, the total wavelength shift becomes smaller. We, therefore, assume, that beside the reversible forward reaction there must be an additional irreversible process. One possible process is the precipitation of the formed carbamate, which led to an increase of the concentration of solved TADDOL. This increase of TADDOL with its high HTP value of $+250 \mu \mathrm{m}^{-1}$ could be the reason for the continuously blue-shift. The data shown in Figure 7 shows additionally that the amplitudes of the shift of each sample during the alternating reactions varied, even if the starting wavelength and the shift of the first forward reaction were similar. One possible explanation is a small diminishing of the degree of orientation in the $\mathrm{N}^{*} \mathrm{LC}$ film during the experiment. The largest blue-shift measured during this experiment was $120 \mathrm{~nm}$. The reverse reaction does not show such a steady shift and reached the equilibrium within minutes.

Another important influence factor was the thickness of the $\mathrm{N}^{*}$ LC film. To keep the electrospun fibers and the nonencapsulated $\mathrm{N}^{*} \mathrm{LC}$ comparable, the layer thickness of the nonencapsulated $\mathrm{N}^{*} \mathrm{LC}$ was adjusted to $3-5 \mu \mathrm{m}$ and controlled by measuring interference spectra. A spectrum with strong interferences and the calculation can be found in Figure S6. Han et al. reported a faster reaction of thicker films and concluded that the reaction is not diffusion controlled. One of the main goals was to investigate the influence of the encapsulation to the response of the sensor and compare it with nonencapsulated $\mathrm{N}^{*} \mathrm{LC}$ applied on a glass surface. The polymer shell, as an additional layer to be permeated by the analyte, should decrease the sensor's shift-rate, while the enlarged surface area should increase it. The shift of the reflection peak maxima due to the reaction with $100 \% \mathrm{CO}_{2}$, as well as the reversible reaction by flushing with synthetic air in alternation every $60 \mathrm{~s}$ is shown in Figure 8. The data was normalized to the maximum value.

A delay of approximately $40 \mathrm{~s}$ between the opening of the MFC-valve and the start of the reaction was seen, which can be explained by dead volume of the used tubing. The reaction of the encapsulated $\mathrm{N}^{*} \mathrm{LC}$ started with a small delay of $2.6 \mathrm{~s}$, as shown in Figure S7. This apparently was the time the $\mathrm{CO}_{2}$

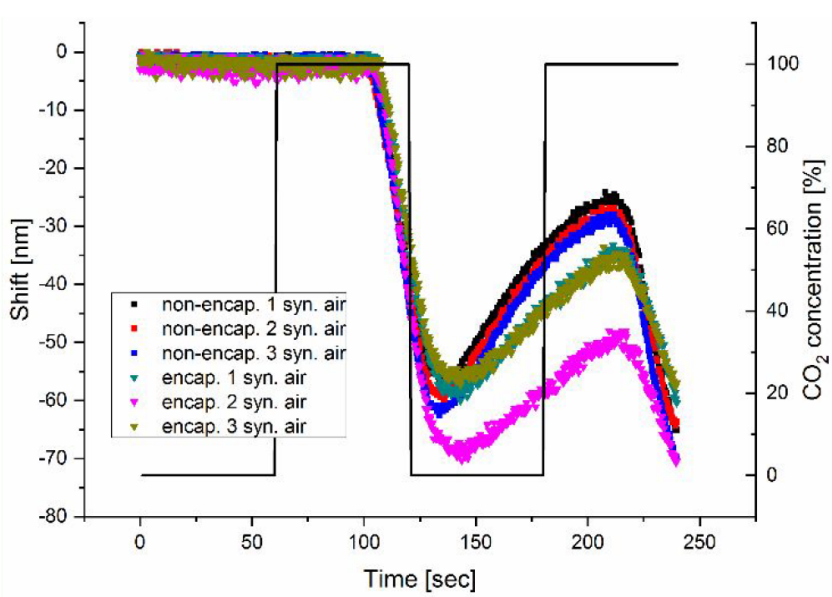

Figure 8. Shift of the reflection maximum while alternating $\mathrm{CO}_{2}$ and synthetic air every $60 \mathrm{~s}$.

needed to permeate the polymer shell. The maximum shift varied between 57-63 $\mathrm{nm}$ for the nonencapsulated and 57-70 for the encapsulated samples. Forming the first-order derivation shows the maximum shift-rates reached and enables a comparison how fast the sensor responses. These results are summarized in Table 1 .

Table 1. Comparison of Maximum Shift Rates When Synthetic Air or Nitrogen Is Used As Purge Gas

\begin{tabular}{|c|c|c|c|}
\hline \multicolumn{4}{|c|}{ maximum shift rates $[\mathrm{nm} / \mathrm{s}]$ using synthetic air } \\
\hline \multicolumn{2}{|c|}{ forward reaction } & \multicolumn{2}{|c|}{ reverse reaction } \\
\hline nonencapsulated & encapsulated & nonencapsulated & encapsulated \\
\hline-2.51 & -2.59 & -0.65 & -0.55 \\
\hline-2.55 & -3.16 & -0.67 & -0.47 \\
\hline-2.92 & -2.59 & -0.73 & -0.49 \\
\hline \multicolumn{4}{|c|}{ maximum shift rates $[\mathrm{nm} / \mathrm{s}]$ using nitrogen } \\
\hline \multicolumn{2}{|c|}{ forward reaction } & \multicolumn{2}{|c|}{ reverse reaction } \\
\hline nonencapsulated & encapsulated & nonencapsulated & encapsulated \\
\hline-3.95 & -3.43 & -0.18 & -0.15 \\
\hline-3.93 & -2.99 & -0.30 & -0.11 \\
\hline-4.09 & -3.71 & -0.29 & -0.18 \\
\hline
\end{tabular}

During the flushing process at the beginning of the experiment no shift of the reflection maximum is observed. This shows that although ethanol appeared to be encapsulated in the fibers there is obviously no evaporation of residual ethanol without heating.

The shift-rates of the forward reaction were nearly identical, while in the reverse reaction a decrease in the shift-rates of the encapsulated samples was seen. The reverse reaction observed here was much faster than in the experiments described by Han et al. It was reported that the reverse reaction of nonencapsulated samples was very slow and a 50\% reconversion of the reaction took $2.7 \mathrm{~h}$ while flushing the sample with pure nitrogen. We observed the same grade of reconversion within minutes when flushing the sample with synthetic air. Apparently, the purge gas applied has a strong influence. A repetition of the experiment using nitrogen (Figure 9) as purge gas indeed shows a significant difference in the response of the sensor.

The comparison in Figure 9 shows, that the maximum shift is much higher and reaches values of $100 \mathrm{~nm}$ within $60 \mathrm{~s}$, when 

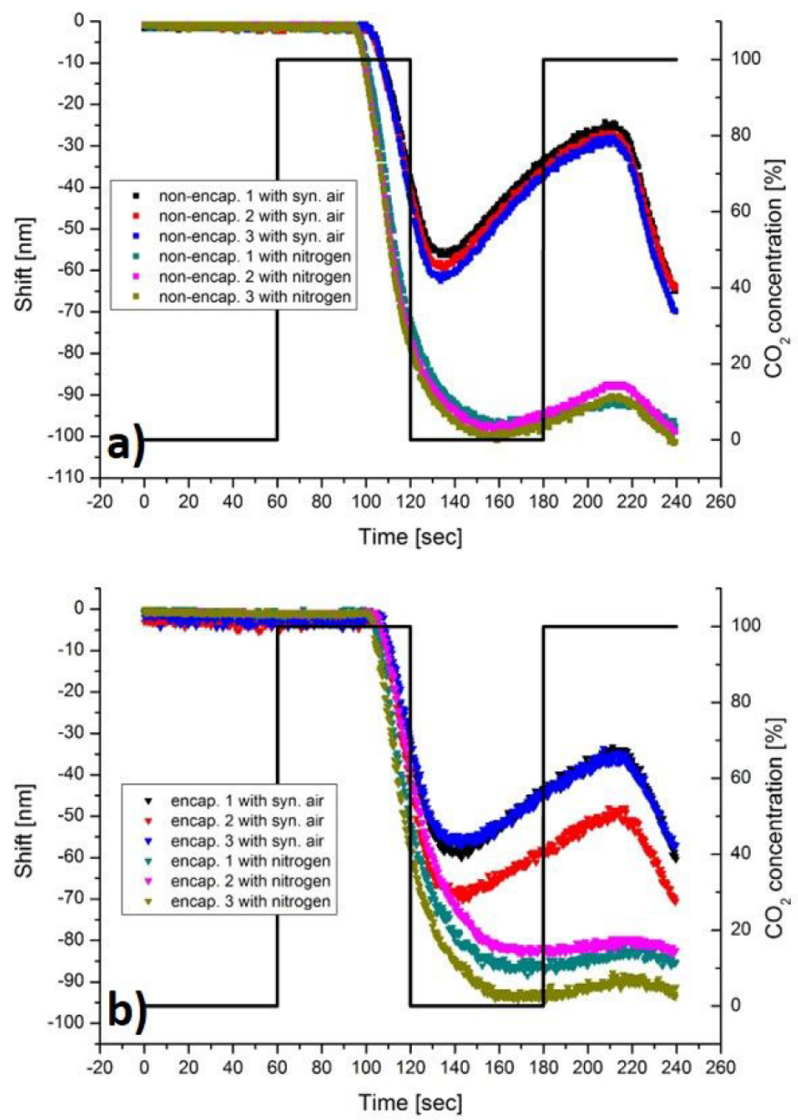

Figure 9. Comparison of (a) nonencapsulated and (b) encapsulated samples using synthetic air and nitrogen as purge gas while alternating $\mathrm{CO}_{2}$ and purge gas every $60 \mathrm{~s}$.

nitrogen is used, which means that the sensor is more sensitive. It is also noteworthy, that the reaction of the nonencapsulated samples started earlier when nitrogen instead of air had been used to flush the sample. Both purge gases were free of water and hydrocarbons, so it seems obvious, that the oxygen in the synthetic air was responsible for this deviant behavior. Up to this point, the authors did not find a chemical reaction, where molecular oxygen influences the formation or cleavage of carbamates. We, therefore, suppose that $\mathrm{O}_{2}$ has a higher solubility in $\mathrm{E} 7$ than $\mathrm{N}_{2}$ and subsequently the diffusion of $\mathrm{CO}_{2}$ into the $\mathrm{N}^{*} \mathrm{LC}$ was hindered. However, higher solubility of $\mathrm{O}_{2}$ can also accelerate the reverse reaction by expelling $\mathrm{CO}_{2}$. The maximum shift rates of nonencapsulated and encapsulated samples when nitrogen was used is summarized in Table 1.

In both, the forward and the reverse reaction the nonencapsulated samples showed slightly higher shift-rates.

\section{CONCLUSION}

We show that an encapsulation of a low-molecular $\mathrm{N}^{*} \mathrm{LC}$ with reactive dopants via coaxial electrospinning in PVP led to a sensor material, which has high potential to improve the applicability of the sensor material used by protecting the fluent $\mathrm{N}^{*}$ LC from environmental influences and giving it a solid form. The sensitivity and shift-rate of the encapsulated $\mathrm{N}^{*} \mathrm{LC}$ was marginally lower, which can be explained by radial orientation of the helical superstructures inside each fiber and the subsequent variety in angles of incidence along the fiber's cross section. However, the orientation of $\mathrm{N}^{*}$ perpendicular to the fiber axis enabled the sensor to be read from all (and not just one selected) viewing angles. A very surprising result was the highly dependent sensitivity on the applied purge gas. When nitrogen was used, a significant higher shift of the reflection peak maximum was observed, while the reverse reaction was hindered. Using synthetic air led to a fast recovery of the sensor within minutes. Encapsulated $\mathrm{N}^{*} \mathrm{LC}$ was found to have a softening-effect to PVP, which decreased the glasstransition temperature by about $100{ }^{\circ} \mathrm{C}$. For a reliable application of this type of sensor, the spinnability of other, water-insoluble polymers should be investigated. At the same time the specific gas permeability of each polymer can be used the improve the selectivity of the sensor. To investigate the relationship between shell thickness and sensitivity, spectroscopic measurements of a single fiber are needed and planned for our further work.

\section{ASSOCIATED CONTENT}

\section{Supporting Information}

The Supporting Information is available free of charge at https://pubs.acs.org/doi/10.1021/acsapm.0c00142.

Molecular structure of hydrogen bonded compound used as $\mathrm{CO}_{2}$-sensitive chiral dopant, photographs of the electrospinning setup with ring-shaped collector electrode, $\mathrm{N}^{*} \mathrm{LC}$ exposed to pure $\mathrm{CO}_{2}$ during GrandjeanCano measurement, reflected wavelength maximum as a function of the sample temperature, DSC curves of uniaxial PVP-fibers and coaxial fibers with $\mathrm{N}^{*} \mathrm{LC}$ in the core, microscopic picture of the inside of the crucible used for coaxial PVP-E7 fibers after the DSC measurement, interferences used for determination of the $\mathrm{N}^{*} \mathrm{LC}$ layer thickness, calculation of $\mathrm{N}^{*} \mathrm{LC}$ layer thickness, and delayed start of the reaction due to the encapsulation and permeation time of the $\mathrm{CO}_{2}$ through the PVP shell (PDF)

\section{AUTHOR INFORMATION}

\section{Corresponding Author}

Lukas Pschyklenk - Institute of Safety and Security Research, University of Applied Sciences Bonn-Rhein-Sieg, 53359 Rheinbach, Germany; (1) orcid.org/0000-0002-2555-9981; Email: Lukas.Pschyklenk@h-brs.de

\section{Authors}

Thorsten Wagner - Department of Chemistry, Paderborn University, 33098 Paderborn, Germany; ○ orcid.org/00000002-4014-0185

Alexander Lorenz - Department of Chemistry, Paderborn University, 33098 Paderborn, Germany; 이이이.org/0000002-6490-7977

Peter Kaul - Institute of Safety and Security Research, University of Applied Sciences Bonn-Rhein-Sieg, 53359 Rheinbach,

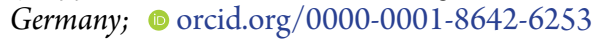

Complete contact information is available at:

https://pubs.acs.org/10.1021/acsapm.0c00142

\section{Notes}

The authors declare no competing financial interest.

\section{ACKNOWLEDGMENTS}

This work is part of the BMBF-Project OptoSpin (FKZ 13FH023IX6). The authors acknowledge the financial support 
by the Federal Ministry of Education and Research of Germany.

\section{REFERENCES}

(1) Brown, G. H.; Shaw, W. G. The Mesomorphic State - Liquid Crystals. Chem. Rev. 1957, 57, 1049-1157.

(2) Castellano, J. A. Liquid Gold: The Story of Liquid Crystal Displays and the Creation of an Industry; World Scientific: Singapore, 2005.

(3) Schenning, A., Crawford, G. P., Broer, D. J., Eds. Liquid Crystal Sensors, The Liquid Crystals Book Series; CRC Press: Boca Raton, FL, 2018.

(4) Fergason, J. L. Liquid Crystals. Sci. Am. 1964, 211, 76-85.

(5) Crissey, J. T.; Bettenhausen, J. M.; Fergason, J. L. Cutaneous Thermography with Liquid Crystals* $\dagger$. J. Invest. Dermatol. 1965, 45, 329-333.

(6) Höller, J. From Machine-to-Machine to the Internet of Things: Introduction to a New Age of Intelligence; Elsevier: Oxford, 2014.

(7) Reinitzer, F. Beiträge zur Kenntniss des Cholesterins. Monatsh. Chem. 1888, 9, 421-441.

(8) Brown, G. H. Advances in Liquid Crystals; Academic Press: New York, 1976.

(9) Mulder, D. J.; Schenning, A. P. H. J.; Bastiaansen, C. W. M. Chiral-nematic liquid crystals as one dimensional photonic materials in optical sensors. J. Mater. Chem. C 2014, 2, 6695-6705.

(10) Kim, D. K.; Hwang, M.; Lagerwall, J. P. F. Liquid crystal functionalization of electrospun polymer fibers. J. Polym. Sci., Part B: Polym. Phys. 2013, 51, 855-867.

(11) Lagerwall, J. P. F.; McCann, J. T.; Formo, E.; Scalia, G.; Xia, Y. Coaxial electrospinning of microfibres with liquid crystal in the core. Chem. Commun. 2008, 5420.

(12) Enz, E.; Lagerwall, J. Electrospun microfibres with temperature sensitive iridescence from encapsulated cholesteric liquid crystal. $J$. Mater. Chem. 2010, 20, 6866.

(13) Scalia, G.; Enz, E.; Calo, O.; Kim, D. K.; Hwang, M.; Lee, J. H.; Lagerwall, J. P. F. Morphology and Core Continuity of LiquidCrystal-Functionalized, Coaxially Electrospun Fiber Mats Tuned via the Polymer Sheath Solution. Macromol. Mater. Eng. 2013, 298, 583589.

(14) Enz, E.; Baumeister, U.; Lagerwall, J. Coaxial electrospinning of liquid crystal-containing poly(vinylpyrrolidone) microfibers. Beilstein J. Org. Chem. 2009, 5, 58.

(15) Enz, E.; La Ferrara, V.; Scalia, G. Confinement-Sensitive Optical Response of Cholesteric Liquid Crystals in Electrospun Fibers. ACS Nano 2013, 7, 6627-6635.

(16) Reyes, C. G.; Sharma, A.; Lagerwall, J. P. F. Non-electronic gas sensors from electrospun mats of liquid crystal core fibres for detecting volatile organic compounds at room temperature. Liq. Cryst. 2016, 43, 1986-2001.

(17) Han, Y.; Pacheco, K.; Bastiaansen, C. W. M.; Broer, D. J.; Sijbesma, R. P. Optical Monitoring of Gases with Cholesteric Liquid Crystals. J. Am. Chem. Soc. 2010, 132, 2961-2967.

(18) Heppke, G.; Oestreicher, F. Notizen: Bestimmung des Helixdrehsinnes cholesterischer Phasen mit der Grandjean-CanoMethode/Determination of the Helical Sense of Cholesteric Liquid Crystals Using the Grandjean-Cano Method. Z. Naturforsch., A: Phys. Sci. 1977, DOI: $10.1515 /$ zna-1977-0818.

(19) Yang, D. K.; Wu, S. T. Fundamentals of Liquid Crystal Devices; Wiley, 2014.

(20) Li, J.; Wen, C.-H.; Gauza, S.; Lu, R.; Wu, S.-T. Refractive Indices of Liquid Crystals for Display Applications. J. Disp. Technol. 2005, 1, 51-61.

(21) Kuball, H.-G.; Weiß, B.; Beck, A. K.; Seebach, D. TADDOLs with Unprecedented Helical Twisting Power in Liquid Crystals. Preliminary communication. Helv. Chim. Acta 1997, 80, 2507-2514.

(22) Teng, J.; Bates, S.; Engers, D. A.; Leach, K.; Schields, P.; Yang, Y. Effect of water vapor sorption on local structure of poly(vinylpyrrolidone). J. Pharm. Sci. 2010, 99, 3815-3825. 